\title{
Double Kernel Method Using Line Transect Sampling
}

\author{
Omar Eidous and M. K. Shakhatreh \\ Department of Statistics, Yarmouk University, Irbid, Jordan
}

\begin{abstract}
A double kernel method as an alternative to the classical kernel method is proposed to estimate the population abundance by using line transect sampling. The proposed method produces an estimator that is essentially a kernel type of estimator use the kernel estimator twice to improve the performances of the classical kernel estimator. The feasibility of using bootstrap techniques to estimate the bias and variance of the proposed estimator is also addressed. Some numerical examples based on simulated and real data are presented. The results show that the proposed estimator outperforms existing classical kernel estimator in most considered cases.

Zusammenfassung: Ein zweifacher Kernschätzer als Alternative zum klassischen Kernschätzer wird vorgeschlagen, um die Artenvielfalt in einer Population zu schätzen, indem man die Stichprobe aus einem linearern Transekt zieht. Die vorgeschlagene Methode ergibt einen Schätzer, der im Wesentlichen vom Typ einer Kernschätzung ist. Nur wird der Kernschätzer zweimal verwendet, um die Eigenschaften des klassischen Kernschätzers dadurch zu verbessern. Auch wird die Möglichkeit angeführt den Bootstrap zu verwenden, um Bias und Varianz des vorgeschlagenen Schätzers zu schätzen. Einige numerische Beispiele basierend auf Simulationen and reellen Daten werden vorgestellt. Die Resultate zeigen, dass der vorgeschlagene Schätzer dem klassischen Kernschätzer in den meisten betrachteten Fällen überlegen ist.
\end{abstract}

Keywords: Line Transect Sampling, Kernel Methods, Boundary Effect, Reflection Method.

\section{Introduction}

Line transect approach to estimate the abundance $D$ of wildlife population has become increasingly popular. In line transect sampling, a strip (a line transect) is surveyed and the perpendicular distances $x_{1}, \ldots, x_{n}$ from the line to the detected objects are recorded. These perpendicular distances are used to model a detection function $g(x)$. This function give us the probability of detecting an object, given that it is at a perpendicular distance $x$ from the line. In addition, the function $g(x)$ help us to find an unbiased estimator for $D$ even if some objects around the transect line are missed. A popular assumption about $g(x)$ is $g(0)=1$, which indicates that there is no missed objects on the transect line, or the probability of detecting an object on the transect line is one. When objects are observed from transect line according to the detection function $g(x)$ then the perpendicular distance $x$ will tend to have a probability density function $f(x)$ of the same shape as $g(x)$ but scaled so that the area under $f(x)$ equals one. The popular fundamental relationship between $f(0)$ and the population abundance is $D=\mathrm{E}(n) f(0) /(2 L)$, where $f(0)=1 / \int_{0}^{\infty} g(x) d x$ under the assumption $g(0)=1, \mathrm{E}(n)$ is the expected value of $n$ (the number of detections) 
and $L$ is the length of the transect line. Therefore the aim of line transect sampling is to estimate $D$ by $\hat{D}=n \hat{f}(0) /(2 L)$ (Burnham, Anderson, and Laake, 1980). Where $\hat{f}(0)$ is an estimator of $f(0)$ based on the observed perpendicular distances $x_{1}, \ldots, x_{n}$ which are assumed to be random sample (Buckland et al., 2001). Hence, the key aspect in line transect sampling is the modeling of $f(x)$ as well as the estimation of $f(0)$. Recent work has focused on employing the nonparametric classical kernel method to estimate $f(0)$. Some initial efforts in applying the kernel method to line transect data were made by Buckland (1992), Chen (1996), Mack and Quang (1998) and Eidous (2005, 2009).

In this paper a new kernel estimator for $f(0)$ is proposed. The proposed estimator is essentially a kernel type of estimator use the kernel estimator twice to improve the performances of the classical kernel estimator. In the next section, we introduce and describe the proposed estimator. Methods to select the bandwidth and the bootstrap technique to compute the approximate bias and variance of the proposed estimator are described in Section 3. Numerical examples are presented in Section 4. Finally, results and conclusions are given in Section 5.

\section{Classical and Double Kernel Estimators}

Let $X_{1}, \ldots, X_{n}$ denote a random sample with common density function $f(x), x \in \mathbb{R}$. The classical kernel density estimator of $f(x)$ (Silverman, 1986) is

$$
\hat{f}_{k}(x)=\frac{1}{n b} \sum_{i=1}^{n} K\left(\frac{x-X_{i}}{b}\right), \quad x \in \mathbb{R},
$$

where $b$ is the smoothing parameter (bandwidth) that controls the degree of smoothness and the kernel function $K$ is assumed to be symmetric probability density function which satisfies $\int u K(u) d u=0$ and $\int u^{2} K(u) d u<\infty$. To reduce the bias (at least asymptotically) of (1), Jones, Linton, and Nielsen (1995) suggested the following double kernel estimator for $f(x)$,

$$
\tilde{f}_{D k}(x)=\frac{\hat{f}_{k}(x)}{n h} \sum_{j=1}^{n} K\left(\frac{x-X_{j}}{h}\right) \hat{f}_{k}^{-1}\left(X_{j}\right), \quad x \in \mathbb{R},
$$

where $h$ is the smoothing parameter not necessary the same as the smoothing parameter $b$ of the classical kernel estimator (1). Jones et al. (1995) derived the theoretical properties of estimator (2). While the classical kernel estimator (1) achieves $O\left(h^{2}\right)$ bias as $h \rightarrow 0$ when $n \rightarrow \infty$, the estimator (2) correct the bias rate to be $O\left(h^{4}\right)$ and the rate of convergence for the variance remains $O\left(n^{-1} h^{-1}\right)$ as the classical kernel estimator.

To use either estimator (1) or (2) for line transect sampling, some modifications are needed because the support of $x$ in this case is defined on the positive real line. This is due to the boundary effects (at $x=0$ ) that occur in nonparametric curve estimation problems (Silverman, 1986). In other words, if our interest is to estimate $f(x)$ at the end point of its support, that is $f(0)$, then $\hat{f}_{k}(0)$ is not even a consistent estimator for $f(0)$. To remove the boundary effect, we adopted the reflection method of Silverman (1986) by applying 
estimators (1) and (2) on the extended sample $X_{1},-X_{1}, X_{2},-X_{2}, \ldots, X_{n},-X_{n}$. By considering the extended sample, the classical kernel estimator of $f(x)$ becomes

$$
\hat{f}_{k 1}(x)=\frac{1}{n b} \sum_{i=1}^{n}\left[K\left(\frac{x-X_{i}}{b}\right)+K\left(\frac{x+X_{i}}{b}\right)\right], \quad 0 \leq x<\infty
$$

and the double kernel estimator of $f(x)$ is suggested to be

$$
\tilde{f}_{D k 1}(x)=\frac{\hat{f}_{k 1}(x)}{n h} \sum_{j=1}^{n}\left[K\left(\frac{x-X_{j}}{h}\right)+K\left(\frac{x+X_{j}}{h}\right)\right] \hat{f}_{k 1}^{-1}\left(X_{j}\right), \quad 0 \leq x<\infty .
$$

Therefore, the classical kernel estimator for $f(0)$ is $\hat{f}_{k 1}(x)$ at $x=0$, which gives

$$
\hat{f}_{k 1}(0)=\frac{2}{n b} \sum_{i=1}^{n} K\left(\frac{X_{i}}{b}\right)
$$

and the double kernel estimator for $f(0)$ is $\tilde{f}_{D k 1}(x)$ at $x=0$, which gives

$$
\tilde{f}_{D k 1}(0)=\frac{2 \hat{f}_{k 1}(0)}{n h} \sum_{j=1}^{n} K\left(\frac{X_{j}}{h}\right) \hat{f}_{k 1}^{-1}\left(X_{j}\right)
$$

where $\hat{f}_{k 1}\left(X_{i}\right)$ and $\hat{f}_{k 1}(0)$ are the classical kernel estimators at $x=X_{i}$ and at $x=0$, respectively. The two quantities $\hat{f}_{k 1}(0)$ and $\hat{f}_{k 1}^{-1}\left(X_{i}\right)$ in estimator (5) are introduced to correct the bias of estimator (4). Jones et al. (1995) pointed out that the leading bias term in $\hat{f}_{k 1}(0)$ should cancel with the leading bias term in $\hat{f}_{k 1}^{-1}\left(X_{i}\right)$. The bandwidth $h$ in estimator (5) is not necessarily the same as the bandwidth $b$ in the estimator (4). Our simulation study in Subsection 4.2 considers the two cases when $h=b$ and when $h$ is not necessarily the same as $b$. Now, the corresponding double kernel estimator for $D$ is

$$
\tilde{D}_{D k 1}=\frac{n \tilde{f}_{D k 1}(0)}{2 L} .
$$

\section{The Bandwidth and some Approximative Properties}

The approximate bias and variance of the proposed estimator can be computed by adopting the bootstrap technique (Efron and Tibshirani, 1993), which is described in the following.

Let $X_{1}, \ldots, X_{n}$ be a random sample of perpendicular distances (collected by using line transect technique) with probability density function $f(x), x \geq 0$, and let $\tilde{f}_{D k 1}(0)$ be the estimator of $f(x)$ at $x=0$ (i.e., $f(0)$ ). To compute the approximative bias and variance of $\tilde{f}_{D k 1}(0)$, a first sample of size $n$ is randomly drawn with replacement from the original sample $X_{1}, \ldots, X_{n}$. The estimator $\tilde{f}_{D k 1}(0)$ is then computed by using this sample and denoted by $\tilde{f}_{D k 1}^{(1)}(0)$. Now, pick a second sample of size $n$ from the original random sample $X_{1}, \ldots, X_{n}$ with replacement and compute $\tilde{f}_{D k 1}^{(2)}(0)$. Repeat the resampling procedure as described above independently some large number $B$ of times, thus 
getting the computed values $\tilde{f}_{D k 1}^{(1)}(0), \ldots, \tilde{f}_{D k 1}^{(B)}(0)$. The approximate bias and variance of $\tilde{f}_{D k 1}(0)$ are given by

$$
\widehat{\operatorname{bias}}\left(\tilde{f}_{D k 1}(0)\right)=\frac{1}{B} \sum_{i=1}^{B} \tilde{f}_{D k 1}^{(i)}(0)-\tilde{f}_{D k 1}(0)
$$

and

$$
\widehat{\operatorname{var}}\left(\tilde{f}_{D k 1}(0)\right)=\frac{1}{B-1} \sum_{i=1}^{B}\left(\tilde{f}_{D k 1}^{(i)}(0)-\frac{1}{B} \sum_{j=1}^{B} \tilde{f}_{D k 1}^{(j)}(0)\right)^{2}
$$

where $\tilde{f}_{D k 1}(0)$ is computed from the original sample $X_{1}, \ldots, X_{n}$.

To implement the proposed estimator in practice we need to choose the value of the bandwidth $h$. One of the most common methods is to find $h$ that minimizing the asymptotic mean integrated square error (AMISE) or the asymptotic mean square error (AMSE) of the estimator. For the classical kernel estimator, Chen (1996) gave the optimal rule for $b=1.06 \hat{\sigma} n^{-1 / 5}$, which minimizes the AMISE of (3) and by considering the half normal distribution to be the underlying distribution of the perpendicular distances. In this setting we adopted two rules to select the bandwidth $h$ of the proposed estimator (5). The first one is $h=b=1.06 \hat{\sigma} n^{-1 / 5}$, which means that the two estimators (4) and (5) use the same level of bandwidth. The corresponding proposed estimator is then denoted by $\tilde{f}_{D k 1}^{*}(0)$. The second rule is called the $m$-nearest neighbor rule, which needs no assumptions about the shape of the perpendicular distances distribution. Barabesi (2001) used this rule in line transect sampling to improve the performance of the local parametric estimator that investigated by Barabesi (2000). Loftsgaarden and Quesenberry (1965) introduced the $m$-nearest neighbor selector in density estimation, which is given by $h=x_{(m)}$, where $x_{(m)}$ represents the $m$ th order statistic in the observed sample. As to the selection of $m$, a common choice is given by $m=\left[\left[n^{\varepsilon}\right]\right]$, where $0<\varepsilon<1$ and $[[\cdot]]$ denotes the greatest integer function. In this setting, we used $\varepsilon=4 / 5$ (see e.g., Mack and Rosenblatt, 1979; Barabesi, 2001. The corresponding proposed estimator that used $h=x_{(m)}$ is denoted by $\tilde{f}_{D k 1}^{* *}(0)$.

\section{Numerical Examples}

\subsection{Wooden Stakes Data}

We apply the proposed estimator to the wooden stakes data set, given in Burnham et al. (1980, p. 61). The data are collected from a line transect survey to estimate the density of stakes in a given area. The stakes data are the perpendicular distances (in meters) of detected stakes from the transect line; 150 stakes were placed at random in an area of $L=1000$ meters long. Out of 150 stakes, 68 stakes were detected using the line transect technique. The true form of $f(x)$ is unknown, but the true value of $f(0)$ is known and equals $f(0)=0.110294$. Thus the actual density of the stakes was $D=n f(0) / 2 L=37.5$ stakes/ha. For comparison purposes we compute the estimators $\hat{f}_{k 1}(0), \tilde{f}_{D k 1}^{*}(0)$ and $\tilde{f}_{D k 1}^{* *}(0)$. The population abundance $D$ is then estimated by $\hat{D}_{k 1}$, 
$\tilde{D}_{D k 1}^{*}$ and $\tilde{D}_{D k 1}^{* *}$ which corresponds $\hat{f}_{k 1}(0), \tilde{f}_{D k 1}^{*}(0)$ and $\tilde{f}_{D k 1}^{* *}(0)$, respectively. The corresponding bias, standard deviation (SD) and mean square error (MSE) for each estimator are computed by using bootstrap technique with 1000 iterations. Careful examination of the data shows the existence of an unusual observation, $x=31.31$, which is too extreme compared to other observations. Therefore, we consider the two sample sizes $n=68$ and $n=67$ separately, the latter sample size is obtained when the outlier observation $x=31.31$ is removed. The two estimators $\hat{f}_{k 1}(0)$ and $\tilde{f}_{D k 1}^{*}(0)$ use the same value of bandwidth which gives $b=1.06 \hat{\sigma} n^{-1 / 5}=3.34$ for $n=67$ (exclude the outlier value $31.31)$ and $b=1.06 \hat{\sigma} n^{-1 / 5}=3.733$ for $n=68$. Accordingly, the resulting $f(0)$ estimates are $\hat{f}_{k 1}(0)=0.1025, \tilde{f}_{D k 1}^{*}(0)=0.1112$ and $\tilde{f}_{D k 1}^{* *}(0)=0.1108$ for $n=67$ with corresponding abundance estimates $\hat{D}_{k 1}=34.33, \tilde{D}_{D k 1}^{*}=37.26$ and $\tilde{D}_{D k 1}^{* *}=37.13$ stakes/ha. The other computations for the two sample sizes and for the different estimators are summarized in Table 1. Burnham et al. (1980) analyze the same data by using a cosine series estimator, and they obtain an estimate for $f(0)$ of 0.1148 with corresponding density estimate $\hat{D}=39.00$ stakes/ha. It should be remarked that the cosine series estimator specifies a fixed value for the maximum perpendicular distance (taken to be 20 meters for this example), that is, more information is used in this case.

Table 1: The point estimates of stakes data abundance (per hectare) and their biases, variances and MSEs. The exact value of $D$ is 0.00375 . Estimator (1) is the classical kernel estimator with $b=1.06 \hat{\sigma} n^{-1 / 5}$, estimator (2) is the double kernel estimator with $h=b=1.06 \hat{\sigma} n^{-1 / 5}$ and estimator (3) is the double kernel estimator with $m$-nearest neighbor method to compute $h$.

\begin{tabular}{ccccccc}
\hline & \multicolumn{6}{c}{$(n=67)$} \\
Estimator & Bandwidth & $\hat{f}(0)$ & $\hat{D}$ & $\operatorname{bias}(\hat{D})$ & $\operatorname{var}(\hat{D})$ & $\operatorname{MSE}(\hat{D})$ \\
\hline 1 & 3.342 & 0.10248 & 34.33 & -0.000301 & $1.464 \cdot 10^{-7}$ & $2.367 \cdot 10^{-7}$ \\
2 & 3.342 & 0.11122 & 37.26 & -0.000024 & $2.226 \cdot 10^{-7}$ & $2.231 \cdot 10^{-7}$ \\
3 & 3.610 & 0.11083 & 37.13 & -0.000005 & $2.001 \cdot 10^{-7}$ & $2.001 \cdot 10^{-7}$ \\
\hline \multirow{7}{*}{ Estimator } & Bandwidth & $\hat{f}(0)$ & $\hat{D}$ & $\operatorname{bias}(\hat{D})$ & $\operatorname{var}(\hat{D})$ & $\operatorname{MSE}(\hat{D})$ \\
\hline 1 & 3.733 & 0.09889 & 33.62 & -0.000377 & $1.451 \cdot 10^{-7}$ & $2.869 \cdot 10^{-7}$ \\
2 & 3.733 & 0.10894 & 37.04 & -0.000058 & $2.157 \cdot 10^{-7}$ & $2.190 \cdot 10^{-7}$ \\
3 & 3.790 & 0.10880 & 36.99 & -0.000021 & $2.177 \cdot 10^{-7}$ & $2.181 \cdot 10^{-7}$ \\
\hline
\end{tabular}

\subsection{Monte Carlo Study}

A simulation study is performed in this section to investigate the performances of the proposed estimators $\tilde{f}_{D k 1}^{*}(0)$ and $\tilde{f}_{D k 1}^{* *}(0)$. The two estimators $\tilde{f}_{D k 1}^{*}(0)$ and $\tilde{f}_{D k 1}^{* *}(0)$ are compared with $\hat{f}_{k 1}(0)$. The half normal bandwidth rule is used to compute $b$ and $h$ for $\hat{f}_{k 1}(0)$ and $\tilde{f}_{D k 1}^{*}(0)$, respectively, while the $m$-nearest neighbor rule is used for $\tilde{f}_{D k 1}^{* *}(0)$. Three families of models which are commonly used as references in line transect studies were considered in the simulation (see Barabesi, 2000, and Eidous, 2009). The exponen- 
tial power (EP) family (Pollock, 1978)

$$
f(x)=\frac{1}{\Gamma(1+1 / \beta)} \exp \left(-x^{\beta}\right), \quad x \geq 0, \beta \geq 1
$$

The hazard-rate (HR) family (Hayes and Buckland, 1983)

$$
f(x)=\frac{1}{\Gamma(1-1 / \beta)}\left(1-\exp \left(-x^{-\beta}\right)\right), \quad x \geq 0, \beta>1
$$

and the beta (BE) model (Eberhardt, 1968)

$$
f(x)=(1+\beta)(1-x)^{\beta}, \quad 0 \leq x<1, \beta \geq 0 .
$$

In our simulation design, these three families were truncated at some distance $w$. Four models were selected from the EP family with parameter values $\beta=1.0,1.5,2.0,2.5$ and corresponding truncation points given by $w=5.0,3.0,2.5,2.0$. Four models were selected from the HR family with parameter values $\beta=1.5,2.0,2.5,3.0$ and corresponding truncation points given by $w=20,12,8,6$. Moreover, four models were selected from the $\mathrm{BE}$ model with parameter values $\beta=1.5,2.0,2.5,3.0$ and $w=1$ for all cases. These models cover a wide range of perpendicular distance probability density functions which vary near zero from a spike to flat. For example, the EP model with $\beta=1$ and the $\mathrm{BE}$ model do not satisfy the shoulder condition (i.e., $f^{\prime}(0) \neq 0$ ). This choice was introduced in order to investigate the robustness of the proposed estimators with respect to the shoulder condition.

For each model and for sample sizes $n=50,100,200$ one thousand runs were iterated. Table 2 reports the relative bias

$$
\mathrm{RB}=\frac{\mathrm{E}[\hat{f}(0)]-f(0)}{f(0)}
$$

and the relative mean error

$$
\mathrm{RME}=\frac{\sqrt{\operatorname{MSE}[\hat{f}(0)]}}{f(0)}
$$

for each considered estimator, where $\hat{f}(0)$ is $\hat{f}_{k 1}(0)$ or $\tilde{f}_{D k 1}(0)$. The efficiency (EFF) of the proposed estimator with respect to the classical kernel estimator is

$$
\mathrm{EFF}=\frac{\operatorname{MSE}\left[\hat{f}_{k 1}(0)\right]}{\operatorname{MSE}\left[\tilde{f}_{D k 1}(0)\right]} .
$$


Table 2: Relative Bias (RB), Relative Mean Error (RME) and Efficiencies (EFF) for the different estimators.

\begin{tabular}{|c|c|c|c|c|c|c|c|c|c|c|}
\hline \multirow[t]{2}{*}{$n$} & \multirow[t]{2}{*}{$\beta$} & \multirow[t]{2}{*}{$w$} & \multicolumn{2}{|c|}{$\hat{f}_{k 1}(0)$} & \multicolumn{2}{|c|}{$\tilde{f}_{D k 1}^{*}(0)$} & \multicolumn{2}{|c|}{$\tilde{f}_{D k 1}^{* *}(0)$} & \multirow[b]{2}{*}{ EFF1 } & \multirow[b]{2}{*}{ EFF2 } \\
\hline & & & $\mathrm{RB}$ & RME & $\mathrm{RB}$ & RME & $\mathrm{RB}$ & RME & & \\
\hline \multicolumn{11}{|c|}{ Exponential Power (EP) Model } \\
\hline 50 & & & -0.3474 & 0.35 & -0.2656 & & -0.2143 & 0.2604 & 253 & 1.382 \\
\hline 100 & 1.0 & 5 & .3302 & & 0.2490 & & -0.2066 & & & 1.461 \\
\hline 200 & & & .2872 & 0.2916 & 0.2072 & & -0.1800 & & .350 & 1.467 \\
\hline 50 & & & 0.1759 & & 0.0927 & & 0.0781 & & 253 & .205 \\
\hline 100 & 1.5 & 3 & -0.1435 & & -0.0643 & & -0.0812 & & 07 & 1.286 \\
\hline 200 & & & -0.1365 & 0.1520 & -0.0683 & & -0.0481 & & 411 & 1.567 \\
\hline 50 & & & .1055 & & 0.0348 & & -0.0157 & & & 1.093 \\
\hline 100 & 2.0 & 2.5 & -0.0762 & 0.1173 & -0.0115 & & -0.0079 & & & 1.030 \\
\hline 200 & & & 515 & & 16 & & 0.0168 & & & 941 \\
\hline 50 & & & .0551 & & 0.0072 & & 0.0167 & & & .952 \\
\hline 100 & 2.5 & 2 & 295 & & 28 & & -0.0035 & & & 811 \\
\hline 200 & & & -0.0262 & & & & 0.0079 & & & .936 \\
\hline \multicolumn{11}{|c|}{ Hazard Rate (HR) Model } \\
\hline 50 & & & -0.5030 & & & & & & & .787 \\
\hline 100 & 1.5 & 20 & .4766 & & -0.4016 & & -0.1 & & 177 & 2.162 \\
\hline 200 & & & -0.4431 & & -0.3623 & & -0.1452 & & & 2.603 \\
\hline 50 & & & & & .2016 & & 446 & & & 750 \\
\hline 100 & 2.0 & 12 & & & -0.1656 & & -0.0456 & & & 2.250 \\
\hline 200 & & & -0.2141 & & -0.1029 & & -0.0223 & & & 2.603 \\
\hline 50 & & & .1608 & & .0515 & & 0.0074 & & & 1.301 \\
\hline 100 & 2.5 & 8 & -0.1132 & & -0.0051 & & 0.0195 & & & 1.379 \\
\hline 200 & & & -0.0805 & & 0.0183 & & 0.0348 & & 97 & 1.340 \\
\hline 50 & & & -0.0641 & & & & & & & 0.880 \\
\hline 100 & 3.0 & 6 & -0.0396 & & 0482 & & 0.0625 & & & 0.765 \\
\hline 200 & & & 265 & & 0.0420 & & 0.0391 & & & .824 \\
\hline \multicolumn{11}{|c|}{ Beta (BE) Model } \\
\hline 50 & & & -0.2105 & & .1444 & & -0.1106 & & & 1.293 \\
\hline 100 & 1.5 & 1 & .1707 & & .1061 & & -0.1081 & & & 1.319 \\
\hline 200 & & & -0.1496 & & -0.0915 & & -0.0831 & & 1.298 & 1.387 \\
\hline 50 & & & & & -0.1321 & & -0.1424 & & 1.250 & 1.165 \\
\hline 100 & 2.0 & 1 & -0.1946 & & -0.1248 & & -0.1241 & & 1.313 & 1.267 \\
\hline 200 & & & -0.1724 & & -0.1089 & & -0.1152 & & 1.332 & 1.338 \\
\hline 50 & & & -0.2459 & & -0.1697 & & -0.1544 & & 1.249 & 1.268 \\
\hline 100 & 2.5 & 1 & -0.2088 & & -0.1367 & & -0.1376 & & 1.321 & 1.302 \\
\hline 200 & & & -0.1920 & 0.2022 & -0.1269 & 0.1501 & -0.1248 & 0.1502 & 1.347 & 1.346 \\
\hline 50 & & & -0.2483 & & & & -0.1639 & & & 1.152 \\
\hline 100 & 3.0 & 1 & -0.2122 & & -0.1361 & & -0.1423 & & 1.333 & 1.293 \\
\hline 200 & & & -0.1955 & 0.2078 & -0.1252 & 0.1524 & -0.1347 & 0.1594 & 1.363 & 1.304 \\
\hline
\end{tabular}




\section{Results and Conclusions}

Depending on the simulation results given in Table 2, we observe that the classical kernel estimator $\hat{f}_{k 1}(0)$ has large $|\mathrm{RB}| \mathrm{s}$ for the EP model with $\beta=1.0,1.5$; for the HR model with $\beta=1.5,2.0$ and for the $\mathrm{BE}$ model with different values of $\beta$. However, its performance is quite good for the EP and HR models when the shape parameter increases, which increases the smoothness of the underlying model near $x=0$. The estimator turns out to be better than $\tilde{f}_{D k 1}^{*}(0)$ and $\tilde{f}_{D k 1}^{* *}(0)$ for the EP model with $\beta=2.5$ and for the HR model with $\beta=3$, in which the efficiencies remain acceptable for these two cases. Both numerical example and simulation results indicate that $\hat{f}_{k 1}(0)$ is underestimated (biases in all cases considered are negative). This motivates the need of the proposed estimators, which are generally produced rather small RBs. On the other hand, comparing the $|\mathrm{RB}| \mathrm{s}$ of $\tilde{f}_{D k 1}^{*}(0)$ and $\tilde{f}_{D k 1}^{* *}(0)$ with that of $\hat{f}_{k 1}(0)$, the simulation and numerical example results generally demonstrate that the $|\mathrm{RB}| \mathrm{s}$ of $\tilde{f}_{D k 1}^{*}(0)$ and $\tilde{f}_{D k 1}^{* *}(0)$ are smaller than the $|\mathrm{RB}| \mathrm{s}$ of $\hat{f}_{k 1}(0)$.

The two estimators $\tilde{f}_{D k 1}^{*}(0)$ and $\tilde{f}_{D k 1}^{* *}(0)$ generally produce reasonable values for RMEs. Their performances are better than that of $\hat{f}_{k 1}(0)$ for most cases considered. A significant improvement for $\tilde{f}_{D k 1}^{*}(0)$ and $\tilde{f}_{D k 1}^{* *}(0)$ over $\hat{f}_{k}(0)$ is clearly demonstrated for the EP model with $\beta=1.0,1.5$; for the HR model with $\beta=1.5,2.0,2.5$ and for the BE model with all different values of $\beta$.

The efficiency (EFF) values show that for most of the models investigated, a considerable gain in the accuracy of the proposed estimators is achieved. Actually, the performances of $\tilde{f}_{D k 1}^{*}(0)$ and $\tilde{f}_{D k 1}^{* *}(0)$ are better than that of $\hat{f}_{k 1}(0)$ for 10 models out of 12 models. In the other two cases where the shoulder condition is - in some sense - large the efficiency is less than one which indicates that the performance of $\hat{f}_{k 1}(0)$ is better than that of $\tilde{f}_{D k 1}^{*}(0)$ and $\tilde{f}_{D k 1}^{* *}(0)$ but the efficiencies remain acceptable in these two cases.

We have seen that the proposed estimators in this paper generally gives small RBs and RMEs. Comparing the classical kernel estimator $\hat{f}_{k 1}(0)$ with the proposed estimators $\tilde{f}_{D k 1}^{*}(0)$ and $\tilde{f}_{D k 1}^{* *}(0)$, the latter two estimators perform better than the former one for most cases considered. Accordingly, the proposed estimators may be recommended in line transect sampling.

\section{Acknowledgements}

We are grateful to the referee and the editor for their suggestions and comments that improved the presentation of this paper.

\section{References}

Barabesi, L. (2000). Local likelihood density estimation in line transect sampling. Environmetrics, 11, 413-422.

Barabesi, L. (2001). Local parametric density estimation methods in line transect sampling. Metron, 59, 21-37.

Buckland, S. T. (1992). Fitting density functions using polynomials. Applied Statistics, 41, 63-76. 
Buckland, S. T., Anderson, D. R., Burnham, K. P., Laake, J. L., Borchers, D. L., and Thomas, L. (2001). Introduction to Distance Sampling. Oxford: Oxford University Press.

Burnham, K. P., Anderson, D. R., and Laake, J. L. (1980). Estimation of density from line transect sampling of biological populations. Wildlife Monograph(72).

Chen, S. X. (1996). A kernel estimate for the density of a biological population by using line-transect sampling. Applied Statistics, 45, 135-150.

Eberhardt, L. L. (1968). A preliminary appraisal of line transects. Journal of Wildlife Management, 32, 82-88.

Efron, B., and Tibshirani, R. (1993). An Introduction to the Bootstrap. New York: Chapman and Hall.

Eidous, O. M. (2005). On improving kernel estimators using line transect sampling. Communications in Statistics: Theory and Methods, 34, 931-941.

Eidous, O. M. (2009). Kernel method starting with half-normal detection function for line transect density estimation. Communications in Statistics: Theory and Methods, 38, 2366-2378.

Hayes, R. J., and Buckland, S. T. (1983). Radial distance models for line-transect method. Biometrics, 39, 29-42.

Jones, M. C., Linton, O., and Nielsen, J. P. (1995). A simple bias reduction method for density estimation. Biometrika, 82, 327-338.

Loftsgaarden, D., and Quesenberry, C. (1965). A nonparametric estimate of multivariate density function. Annals of Mathematical Statistics, 36, 1049-1051.

Mack, Y. P., and Quang, P. X. (1998). Kernel methods in line and point transect sampling. Biometrics, 54, 606-619.

Mack, Y. P., and Rosenblatt, M. (1979). Multivariate $k$-nearest neighbor density estimates. Journal of Multivariate Analysis, 9, 1-15.

Silverman, B. W. (1986). Density Estimation. London: Chapman and Hall.

\author{
Authors' addresses: \\ Omar Eidous and M. K. Shakhatreh \\ Department of Statistics \\ Yarmouk University \\ Irbid \\ Jordan \\ E-mails: omarm@yu .edu .jo, moh.shakhatreh@yu .edu .jo
}

